\title{
NWPEsSe: an Adaptive-Learning Global Optimization Algorithm for Na- nosized Cluster Systems
}

\author{
Jun Zhang, Vassiliki-Alexandra Glezakou* Roger Rousseau, Manh-Thuong Nguyen \\ Physical Sciences Division, Pacific Northwest National Laboratory, Richland, WA, 99352, United States
}

\begin{abstract}
Global optimization constitutes an important and fundamental problem in theoretical studies in many chemical fields, such as catalysis, materials or separations problems. In this paper, a novel algorithm has been developed for the global optimization of large systems including neat and ligated clusters in gas phase, and supported clusters in periodic boundary conditions. The method is based on an updated artificial bee colony $(\mathrm{ABC})$ algorithm method, that allows for adaptive-learning during the search process. The new algorithm is tested against four classes of systems of diverse chemical nature: gas phase $\mathrm{Au}_{55}$, ligated $\mathrm{Au}_{8}^{2+}, \mathrm{Au}_{8}$ supported on graphene oxide and defected rutile, and a large cluster assembly $\left[\mathrm{Co}_{6} \mathrm{Te}_{8}\left(\mathrm{PEt}_{3}\right)_{6}\right]\left[\mathrm{C}_{60}\right]_{n}$, with sizes ranging between 1 to $3 \mathrm{~nm}$ and containing up to 1300 atoms. Reliable global minima (GMs) are obtained for all cases, either confirming published data or reporting new lower energy structures. The algorithm and interface to other codes in the form of an independent program, Northwest Potential Energy Search Engine (NWPEsSe), is freely available and it provides a powerful and efficient approach for global optimization of nanosized cluster systems.
\end{abstract}

\section{Introduction}

Chemical clusters are aggregates consisting of a couple of to a few thousand structural units like atoms or molecules. They are increasingly capturing attention from the chemical community because they exhibit unique properties in electronic structure, ${ }^{1}$ magnetics, ${ }^{2}$ geometry, ${ }^{3}$ catalysis, ${ }^{4}$ and energy storage. ${ }^{5}$ In addition, being a bridge between a few atoms and bulk matter, chemical clusters make good models for computational studies of realistic and complicated problems. For example, using a solute-solvent cluster to accurately calculate the hydration energies of metallic cations ${ }^{6,7}$ or to study chemical reaction mechanisms. ${ }^{8}$

The determination of the global minimum (GM) of a chemical cluster on its potential energy surface (PES) is the first step towards the study of a system. At low temperature, the GM usually dominates over other structures of similar energy. Also, GM suggest the most favorable interaction pattern between the atoms or structural units in the chemical cluster, revealing valuable chemical information. Searching for the GM is much more difficult than locating a local minimum (LM), since it requires the knowledge over the whole PES instead of just a small region. Chemists have been working on developing effective global optimization algorithms for decades. Examples of such methods rely on simulated annealing, ${ }^{9}$ Monte Carlo minimization, ${ }^{10}$ basin hopping, ${ }^{11-15}$ genetic algorithms, ${ }^{16-23}$ particle swarm optimization, ${ }^{24}$ stochastic surface walking, ${ }^{25}$ kick method, ${ }^{26-29}$ GIGA, ${ }^{30}$ and have shown good performance for various chemical problems. We also refer the readers to reviews ${ }^{31,32}$ or our previous papers ${ }^{33,34}$ for a comprehensive discussion.

Since 2004, instead of empirical force-fields, reliable first-principles methods began to appear for calculating cluster energies. The earliest global optimizations at the density functional theory (DFT) level of theory for gas phase and surface-supported clusters were carried out for $\mathrm{Na}_{n} \mathrm{Cl}_{n+1}^{-}$in $2004^{35}$ and $\mathrm{Ag}_{1 \sim 4,6,8,10} @ \mathrm{MgO}(100)$ in 2007, ${ }^{36}$ respectively. Examples of global optimization algorithms used on a variety of systems (aperiodic or periodic) have been reported in the literature and include $\mathrm{Li}_{n}^{q},{ }^{37} \mathrm{Ir}_{n},{ }^{38} \mathrm{Pt}_{n},{ }^{39}$ graphene-supported $\mathrm{Pt}_{n},{ }^{40} \mathrm{MgO}$ supported $\mathrm{AuPd},{ }^{41} \mathrm{TiCl}_{4}$-capped $\mathrm{MgCl}_{2}$ plate (Ziegler-Natta catalyst $),{ }^{42}$ to name a few. Other general-purpose codes include PDECO,${ }^{43}$ GEGA,${ }^{37}$ GMIN, ${ }^{44}$ TGMIN,${ }^{45}$ AUTOMATON,${ }^{46}$ etc. However, some of these codes are either not readily available, lack the interface to common computational chemistry programs, or are simply designed for specific systems.

Compared with other global optimization methods, the artificial bee colony (ABC) algorithm, which was proposed in 2005 in the computer science community, ${ }^{47}$ is simple but effective. We adapted the algorithm for chemical problems and wrapped it in a program called ABCluster. ${ }^{33,}{ }^{34}$ Since then, ABCluster has gained wide applications in chemical problems from different fields. One example is that ABCluster was used to search the GMs of lanthanide ( $\mathrm{Ln}$ ) doped silicon cluster anions as large as $\mathrm{LnSi}_{20}^{-}$, and the calculated photoelectron spectroscopy from these GMs agree well with the experimental ones. ${ }^{4-57}$ Another example is that several authors searched the GMs of clusters consisting of atmosphere related molecules like water, ammonia, nitric acid, hydroxymethanesulfonic acid, sulfuric anhydride, and pentoxide-iodic acid. ${ }^{58-70}$ Using GMs of different sizes, atmospheric cluster dynamics equations can be solved ${ }^{71}$ and a lot of atmospheric chemical phenomena were successfully explained. ${ }^{58-70}$ ABCluster was also applied in catalysis, ${ }^{4,}$ 72-74 material science, ${ }^{75-77}$ solution chemistry, ${ }^{78-80}$ etc. Therefore, the $\mathrm{ABC}$ algorithm is proven to be a highly successful method for searching GMs of atomic and molecular clusters to solve realistic chemical problems.

While clusters of atoms and molecules can be used to solve a lot of chemical problems as described above, more complicated clusters are desired in state-of-the-art researches. For example, in electrochemical catalysis, surface-supported metallic clusters play an important role in many chemical processes. ${ }^{81,82}$ In the study of energy chemistry or soft matter, very large (nanosized) 
clusters of molecules of different chemical properties, which could be of heterogeneous structure, are needed for theoretical insights.

In this paper, we propose a new algorithm, Northwest Potential Energy Surface Search Engine (NWPEsSe) based on the ABC method, for the efficient global optimization of nanosized clusters. Several systems of distinct chemical nature are used to demonstrate the efficiency of this newly developed algorithm, showing its ability in obtaining reliable structures of highly complex gas phase, ligated, surface-supported, and cluster assemblies containing more than 1300 atoms. We anticipate that its flexibility and efficiency will make it a useful tool for many scientists in different fields.

\section{Method}

The method will be discussed in detail in this section. The algorithm discussed below has been coded into NWPEsSe and can be downloaded free of charge from https://store.pnnl.gov/catalog-products/open-source .

\subsection{Coordinate Systems}

The structural units of a cluster can be atoms, small molecules, microcrystals, or surface slabs. They are defined by a set of Cartesian coordinates (in a body-fixed coordinate system). To build an initial structure during the global optimization, these structural units are manipulated as rigid bodies, i.e., their internal degrees of freedom (DOFs) are frozen (The internal DOFs will be relaxed in their local optimization step). Therefore, the coordinate of each structural unit is represented by 6-component vector $\mathbf{q}=\{\mathbf{R}, \mathbf{\Omega}\}$ : the geometrical center $\mathbf{R} \equiv\{X, Y, Z\}$, which determines its position, and the Euler angles $\boldsymbol{\Omega} \equiv\{\alpha, \beta, \gamma\}$, which determines its orientation. This representation has been used in our previous study ${ }^{34}$ and proves to be highly successful. Their Cartesian coordinates in the cluster (in a space-fixed coordinate system) can be calculated using ${ }^{34}$

$$
\left(\begin{array}{c}
x_{\text {space }} \\
y_{\text {space }} \\
z_{\text {space }}
\end{array}\right)=\mathbf{R}_{z}(\gamma) \mathbf{R}_{y}(\beta) \mathbf{R}_{z}(\alpha)\left(\begin{array}{l}
x_{\text {body }} \\
y_{\text {body }} \\
z_{\text {body }}
\end{array}\right)+\left(\begin{array}{c}
X \\
Y \\
Z
\end{array}\right)
$$

where

$$
\begin{aligned}
& \mathbf{R}_{z}(\theta) \equiv\left(\begin{array}{ccc}
\cos \theta & -\sin \theta & 0 \\
\sin \theta & \cos \theta & 0 \\
0 & 0 & 1
\end{array}\right) \\
& \mathbf{R}_{y}(\theta) \equiv\left(\begin{array}{ccc}
\cos \theta & 0 & \sin \theta \\
0 & 1 & 0 \\
-\sin \theta & 0 & \cos \theta
\end{array}\right)
\end{aligned}
$$

Therefore, a cluster consisting of $N$ structural units will be represented by $6 N$ components: $\mathbf{Q} \equiv\left\{\mathbf{q}_{1}, \mathbf{q}_{2}, \cdots, \mathbf{q}_{N}\right\}$.

\subsection{Generation of Random Structures with Given Features}

In the previous implementation of $\mathrm{ABC}$ in ABCluster for simple atomic and molecular clusters, ${ }^{33,34}$ the initial structure of a cluster is generated completely randomly. This is a reasonable approach since the structures of small clusters are likely to be more disordered and therefore more difficult to predict. In addition, mixed clusters, can adopt structures that are drastically different from those of pure species. For example, when metal atomic interactions are described with the Gupta potential, ${ }^{83}$ both $\mathrm{Ag}_{38}$ and $\mathrm{Cu}_{38}$ are truncated octahedra. However, the mixed cluster $\mathrm{Ag}_{32} \mathrm{Cu}_{6}$ has an unexpected copper-core-silvershell structure. ${ }^{84}$ Clearly, as the complexity of a system increases, either because of its composition or its size, a different sampling seems to be necessary. Sampling of nanosized clusters, especially heterogeneous ones, can be extremely inefficient since part of the system may still maintain order that random searches may skip over.

NWPEsSe was developed to address some of these issues by utilizing the power of the ABC algorithm augmented by adaptive-learning techniques, where the population of structures on the PES increases with acceptable ('good') structures along the way. This new approach effectively uses several new features to accelerate the global optimization, exemplified by two typical examples:

(1) Surface-supported clusters. For a surface slab or microcrystal structure, structural units can be identified as parts of a surface with specified atoms/molecules pointing toward specific contact sites of the surface. In NWPEsSe surfaces can be detected automatically by calculating its convex hull ${ }^{85}$ of a finite set of points.

(2) Shell-core structures. When some structural units tend to bond to or point towards a core, as for example ligated clusters, they can be distributed uniformly or randomly on an ellipsoid shell of specified size and orientation. A uniform distribution is determined by solving Thomson's problem ${ }^{86}$ where we look for a minimum electrostatic potential energy configuration of electrons constrained to a unit sphere and then map the solutions to an ellipsoid, see Figure 1 and supporting information for more details

Besides these two features, NWPEsSe also features several other options, for example, distribute structural units inside a box or a sphere. These features can be combined to control the possible cluster structures. In the following sections, all references of "random structure" refer to any random cluster generated with given features.

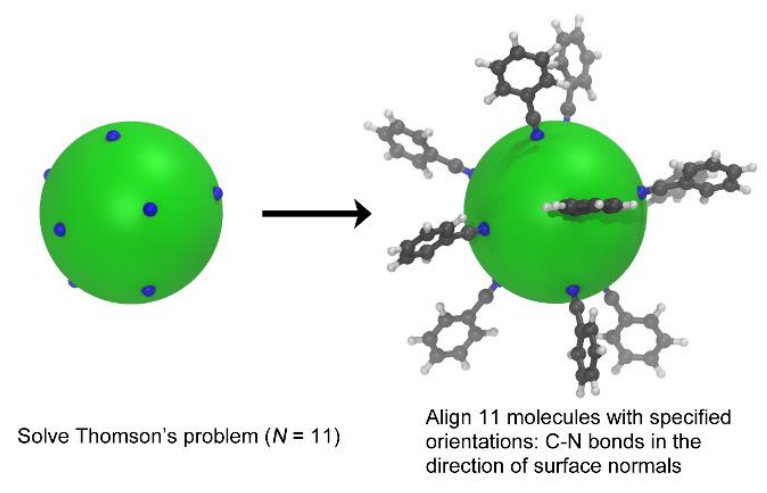

Figure 1. Generation of a shell-core structure of 11 benzonitrile molecules.

\subsection{Local Optimization of Clusters}

Once a guess structure is generated, it is submitted to a quantum chemistry program for local geometry optimization and energy calculation. In NWPEsSe, the guess structure is pre-relaxed to remove unreasonable structural motifs, such as short interatomic distances, that could significantly increase the optimization steps or lead to convergence problems. This is achieved by defining a potential:

$$
u\left(r_{i j}\right)=\left\{\begin{aligned}
k\left(r_{i j}-r_{i}^{\mathrm{c}}-r_{j}^{\mathrm{c}}\right)^{2}, & r_{i j}<r_{i}^{\mathrm{c}}+r_{j}^{\mathrm{c}} \\
0, & r_{i j} \geq r_{i}^{\mathrm{c}}+r_{j}^{\mathrm{c}}
\end{aligned}\right.
$$


where $r_{i} / r_{j}$ is the distance between atom $i$ and $i, r_{i}^{\mathrm{c}} / r_{j}^{\mathrm{c}}$ their covalent radii, and $k$ the force constant (see Figure S1). The complete potential for the cluster is then constructed as:

$$
U(\mathbf{Q})=\sum_{I}^{\substack{\text { structural } \\ \text { units }}} \sum_{J<I}^{\substack{\text { structural } \\ \text { units }}} \sum_{i_{I} \in I} \sum_{j_{J} \in J} u\left(r_{i_{I} j_{J}}\right)
$$

where, $I$ and $J$ are indices of structural units, and $i_{I}$ are $j_{J}$ indices of the atoms in $I$ and $J$, respectively. We optimize eq. (5) with respect to $\mathbf{Q}$ using the limited-memory-BroydenFletcher-Goldfarb-Shanno (L-BFGS) algorithm ${ }^{87}$. During this process, the structural units will be pushed away from others until any two atoms from different structure units are farther away than the sum of their covalent radii. The resulting cluster is free of atomic clashes and can be safely optimized with other quantum chemical programs, see Figure 2.

NWPEsSe can also be used as a fast structure generator even without further optimization by external programs, since the structure defined by eq. (5) is already very reasonable as an initial guess for quantum chemical calculations or molecular dynamics simulations. Using the structural features provided, complicated clusters can be generated rapidly, see Supporting Information or NWPEsSe Manual for more details.

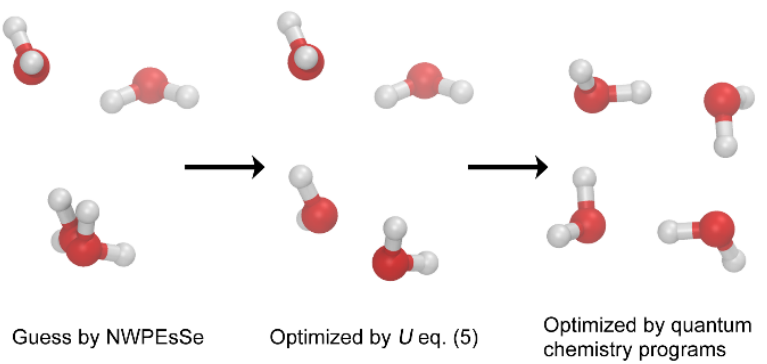

Figure 2. The local optimization of a guess cluster structure.

\subsection{The Artificial Bee Colony Algorithm}

The ABC algorithm is a swarm intelligence-based algorithm, mimicking the foraging behavior of honey bee colonies. ${ }^{47}$ In nature, bees search for the best nectar as a food source using an efficient strategy conducted by three kinds of bees: (1) Employed bees (EM), which explore surroundings; (2) Onlooker bees (OL), which search for the "good" nectars reported by EM; (3) Scout bees (SC), which explore completely new environments. In our implementation, clusters and their energies correspond to the nectars and their quality where lower energies imply "better" nectars. This is the basic framework of the algorithms used in both ABCluster and NWPEsSe. The discussion below will focus on the differences between the two algorithms. For more details on ABCluster, please refer to previous papers. ${ }^{33,34}$

The main steps of the NWPEsSe algorithm can be summarized as:

(1) Initialization: the structure population size $S N$, the maximum cycle number $g_{\text {max }}$, cycle number $g=0$;

(2) Generate $S N$ random structures and optimize them using quantum chemistry, obtaining the structures $\mathbf{Q}_{1}^{0}, \mathbf{Q}_{2}^{0}, \cdots, \mathbf{Q}_{S N}^{0}$ and their energy $E_{1}^{0}, E_{2}^{0}, \cdots, E_{S N}^{0}$;
(3) At cycle $g$, the guess structure is generated using one of the three ways below with equal probability:

(3.1) EM: a guess structure is formed using the trigonometric mutation operator: ${ }^{88}$

$$
\begin{aligned}
& \mathbf{Q}=\frac{1}{3}\left(\mathbf{Q}_{k_{1}}^{g}+\mathbf{Q}_{k_{2}}^{g}+\mathbf{Q}_{k_{3}}^{g}\right)+\left(p_{2}-p_{1}\right)\left(\mathbf{Q}_{k_{1}}^{g}-\mathbf{Q}_{k_{2}}^{g}\right) \\
& +\left(p_{3}-p_{2}\right)\left(\mathbf{Q}_{k_{2}}^{g}-\mathbf{Q}_{k_{3}}^{g}\right)+\left(p_{1}-p_{3}\right)\left(\mathbf{Q}_{k_{3}}^{g}-\mathbf{Q}_{k_{1}}^{g}\right)
\end{aligned}
$$

where $k_{1}, k_{2}, k_{3}$ are random integers between 1 and $S N\left(k_{1} \neq\right.$ $k_{2} \neq k_{3} \neq i$ ), and

$$
p_{m}=\frac{\left|E_{k_{m}}^{g}\right|}{\left|E_{k_{1}}^{g}\right|+\left|E_{k_{2}}^{g}\right|+\left|E_{k_{3}}^{g}\right|} \quad(m=1,2,3)
$$

The obtained structure by eq. (6) can preserve the given features since it is a deformed average structure of three clusters. The EM bee step performs an exploration in a new direction in the parameter space based on a triple of individuals. Eq. (6) has been proven to be highly efficient in exchanging information between individuals of a population in swarm-intelligencebased algorithms, ${ }^{33,34,43,88}$ introducing multiple interactions between individuals. This is a random exploration of cluster structures.

(3.2) OL: a guess structure is formed by deformation of the current best cluster (i.e., of lowest energy) $\mathbf{Q}_{\text {best }}^{g}$ using the $\mathrm{ABC} /$ best $/ 2$ strategy: ${ }^{33}$

$$
\mathbf{Q}=\mathbf{Q}_{\text {best }}^{g}+F\left(\mathbf{Q}_{k_{1}}^{g}+\mathbf{Q}_{k_{2}}^{g}-\mathbf{Q}_{k_{3}}^{g}-\mathbf{Q}_{k_{4}}^{g}\right)
$$

where $k_{1}, k_{2}, k_{3}, k_{4}$ are random integers between 1 and $S N$ $\left(k_{1} \neq k_{2} \neq k_{3} \neq k_{4}\right)$ and $F$ is a random real number in $[0,1)$. The structure by eq. (8) can be viewed as a perturbation of the current GM like the moving step in Monte-Carlo methods. This $\mathrm{ABC} / \mathrm{best} / 2$ strategy was originally used in the differential evolution algorithm ${ }^{89}$ but was also proved to be effective in the ABC algorithm. ${ }^{90}$ Since the OL bee step is an exploration based on "good" individuals, it gives a positive feedback in the optimization process. This step adds a bias in the search toward good structures.

(3.3) SC: in the population, if a cluster has a very high energy compared to a given cutoff that can be defined by the user, or a structure similar to others, it will be replaced by a random structure. In this case, the SC bee step is set against the "bad" individuals, forming a negative feedback. This step prevents the search from being trapped in a local minimum basin.

(4) The guess structure $\mathbf{Q}$ is optimized using quantum chemical programs. The optimized structure is added to the population, which increases with 'good' structures as the search progresses.

(5) If $g \leq g_{\text {max }}$, increase $g$ by 1 and go back to step (3); otherwise the search is finished.

Figure 3 provides the basic steps of the NWPEsSe algorithm (A), the adaptive part (B) and finally a side-by-side comparison with ABCluster in the generation of the polulations (C). We note two significant difference from ABCluster:

(1) in ABCluster, in each step all three types of bees, EM, OL, and SC, operate on the entire population. This step would be too expensive for first-principles global optimization of large structures. Thus, in NWPEsSe, only one kind of bee (EM or OL or SC) is applied during each cycle, but with equal probability $(1 / 3)$. This is a trade-off between computational cost and selection efficiency. 
(2) However, the performance of the search is offset by the second difference: the population is extended with new guess clusters instead of being kept constant. As the population expands, NWPEsSe will have a cluster structure library of higher diversity and quality. Through this adaptive learning process described by Eqs. (6) and (8), the bees become more capable of discovering improved cluster structures. As shown in Figure 3, the algorithm contains fluctuations (random structures), multiple interactions (EM bees), and positive (OL bees) and negative
(SC bees) feedbacks. The four features lead to a self-organizing $^{91}$ algorithm. Self-organization provides a mechanism for an adaptive learning procedure during global optimization, keeping the diversity of the population without being trapped in a local minimum basin. The efficiency of the new algorithm in NWPEsSe is demonstrated by the select systems discussed below.
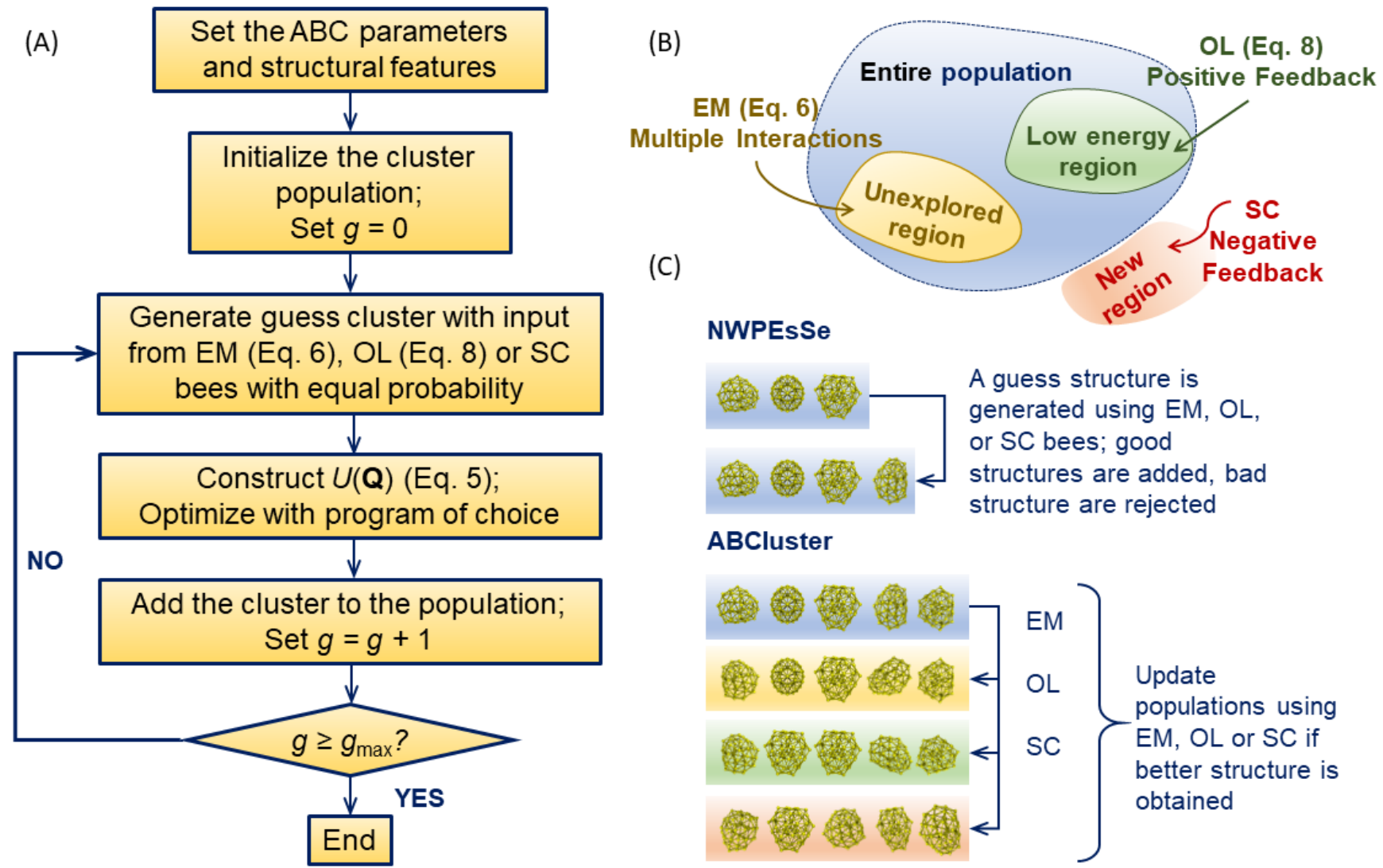

Figure 3. (A) The NWPEsSe algorithm. (B) Schematic of population update based on evaluation by EM, OL and SC bees. (C) Comparison between the NWPEsSe and ABCluster algorithms: In NWPEsSe, all types of bees are operating on the population, updating with 'good' structures, while rejecting 'bad' ones based on positive and negative feedback. Over time, the population becomes larger with 'good' structures, creating an adaptive learning environment. In ABCluster, each group of bees operates serially on the fixed population, with random updates of the population.

\subsection{Quantum Chemical Calculation Details}

NWPEsSe also provides interfaces to a variety of computational chemistry programs. In this paper, two programs were used for the tests performed: $\mathrm{xTB}$ and $\mathrm{CP} 2 \mathrm{~K}$. xTB is a program implementing the highly versatile GFN-xTB Hamiltonian, ${ }^{92,}{ }^{93}$ which is a fast semi-empirical method for molecules. $\mathrm{CP} 2 \mathrm{~K}^{94,95}$ is used for both gas phase and condensed phase DFT calculations and molecular dynamics simulations. In all DFT calculations, the gradient corrected functional $\mathrm{PBE}^{96}$ with Grimme dispersion correction DFT-D3, ${ }^{97}$ in the $\Gamma$-point approximation were employed for Brillouin zone integration. A molecularly optimized double- $\zeta$ Gaussian basis ${ }^{98}$ and plane wave basis ${ }^{99}$ of 420 Ry cutoff were applied to all the atoms, while core electrons were represented by the companion norm-conserving pseudopotentials. ${ }^{100,101}$

\section{Applications}

It should be emphasized that we selected systems inspired by our research from our BES Catalysis and Separations programs which also demonstrate the versatility and performance of our algorithm.

\subsection{Gas Phase Gold Clusters}

Gold nanoparticles exhibit excellent catalytic and optical properties and therefore have a broad spectrum of applications. ${ }^{102}$ While the GMs of small gold clusters (up to 20 atoms) are well characterized, ${ }^{103}$ the GM of larger gold cluster are still under investigation. Experimental determination of their structures is difficult. A typical example is the $1.1 \mathrm{~nm}$ cluster $\mathrm{Au}_{55}$, the $\mathrm{GM}$ of which has been the topic of several studies. ${ }^{104,105}$

NWPEsSe was used in the search of the GM of $\mathrm{Au}_{55}$. The local optimization was carried out with xTB using $g_{\max }=3000$, then the 30 lowest energy clusters were further optimized at the 
DFT level of theory with CP2K. The cluster with the lowest energy, obtained from this search encompassing 3000 semiempirical and 30 DFT calculations, was taken as the putative GMs. Besides this, the GMs proposed in two previous papers by Tarrat et al ${ }^{105}$ and van den Bossche ${ }^{104}$ as well as an icosahedral isomer were also optimized using DFT for comparison. Three functionals: $\mathrm{PBE},{ }^{96}$ revPBE, ${ }^{106}$ and PBEsol ${ }^{107}$ were used. The $\mathrm{CP} 2 \mathrm{~K}$ calculations were carried out in gas phase in a periodic a $2 \times 2 \times 2 \mathrm{~nm}$ cell affording at least 1 nm space between a cluster and its periodic image.

The results are given in Table 1 and Figure 4. The energies of GMs predicted with NWPEsSe Au55-0 and by van den Bossche $^{104}$ Au55-1 are essentially identical. The GM by Tarrat et $a l^{105}$ Au55-2 has $D_{3}$ symmetry, is predicted to be a high-lying isomer by $\mathrm{PBE}$ and PBEsol, but comparable in stability with
Au55-0 or Au55-1 as indicated by revPBE. The icosahedral isomer Au55-3 has the highest symmetry as well as the highest energy. Since low-coordinated gold atoms (surface ones) tend to form short bonds, ${ }^{108}$ the inner-shell atoms in icosahedral Au55-3 migrate to the outer-shell, resulting in shorter (by about $0.01 \mathrm{~nm}$ ) surface Au-Au distances and lower energy. As a result, the cluster loses its $I_{\mathrm{h}}$ symmetry, resulting in disordered but more stable isomers. Interestingly, while Au55-0 and Au55-1 have similar energies and are both disordered with a two-shell 46-9 geometry, their inner shells are quite different (Figure 5), implying that the low energy part of the $\mathrm{Au}_{55} \mathrm{PES}$ is flat and contains several disordered structures. This is in agreement with direct atomic imaging experiments by Wang et al, ${ }^{109}$ where no highly symmetrical structures of $\mathrm{Au}_{55}$ were observed.

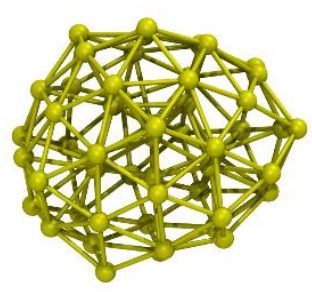

Au55-0

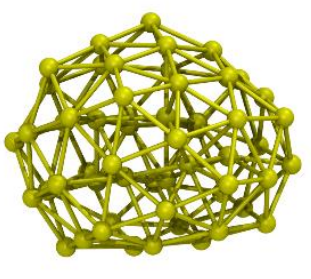

Au55-1

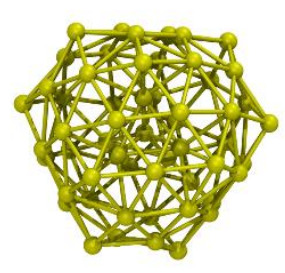

Au55-2

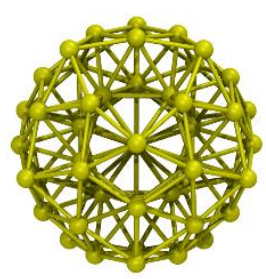

Au55-3

Figure 4. The isomers of $\mathrm{Au}_{55}$ optimized at PBE level of theory. Au55-0 is the one found by NWPEsSe.
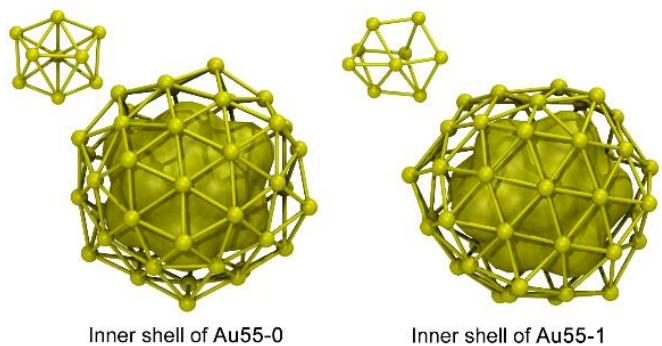

Inner shell of Au55-1

Figure 5. Inner shells of Au55-0 and Au55-1.

Table 1. Relative energies (Unit: kcal mol $\mathbf{~}^{-1}$ ) of isomers of $\mathrm{Au}_{55}$.

\begin{tabular}{lllll}
\hline Isomer & Symmetry & PBE & revPBE & PBEsol \\
\hline Au55-0 & $C_{1}$ & 0.00 & 0.00 & 0.00 \\
Au55-1 & $C_{1}$ & 0.10 & 0.01 & 0.30 \\
Au55-2 & $D_{3}$ & 12.84 & 0.72 & 35.94 \\
Au55-3 & $I_{\mathrm{h}}$ & 29.32 & 31.19 & 22.27 \\
\hline
\end{tabular}

\subsection{Ligated Gold Clusters}

Metal clusters play an important role in catalysis and separations, and organic ligands are often used to stabilize their structure and tune their catalytic ${ }^{110,111}$ or steric properties. ${ }^{112,113}$ Here, we consider the ligated gold cluster cation $\mathrm{Au}_{8} \mathrm{~L}_{n}^{2+}$, where $\mathrm{L}$ is a simple phosphine ligand $\mathrm{P}\left(\mathrm{CH}_{3}\right)_{3}$.

Here, we started also with xTB to search for the GMs of $\mathrm{Au}_{8} \mathrm{~L}_{n}^{2+}$ using $g_{\max }=500$. Next, 30 lowest energy clusters were further optimized at the PBE-D3 level of theory with CP2K. The most stable cluster cation ranked by PBE-D3 was taken as the putative GM. In total, 500 semi-empirical and 30 DFT calculations were used to determine the GM for each system. These $\mathrm{CP} 2 \mathrm{~K}$ calculations were carried out in gas phase in a $2.5 \times 2.5 \times 2.5 \mathrm{~nm}$ cell.

The results are shown in Figure 6. For the bare cluster cation, the predicted GM Au8c-0 is by $4.12 \mathrm{kcal} \mathrm{mol}^{-1}$ more stable than Au8c-1, which was proposed as a GM by Hong et al. ${ }^{114}$ Interestingly, for $\mathrm{Au}_{8} \mathrm{~L}_{n}^{2+}$ where $\mathrm{L}=\mathrm{P}\left(\mathrm{CH}_{3}\right)_{3}$, when $n=4$ and 5, their GMs have Au8c-1 as their cores. When the cores were substituted by Au8c-0, in the optimized cluster cations (Au8cL4-1 and Au8cL5-1), the initial Au8c-0 core relaxed to another structure and are overall less stable than their GMs by 4.98 and $0.39 \mathrm{kcal} \mathrm{mol}^{-1}$, respectively; As $n$ goes to 6,7 , and 8, the cores of GMs (Au8cL6-0, Au8cL7-0, Au8cL8-0) change from flake to polyhedron. For example, one $\mathrm{LM}$ of $\mathrm{Au}_{8} \mathrm{~L}_{8}^{2+}$ Au8cL8-1 with a flake core is $8.32 \mathrm{kcal} \mathrm{mol}^{-1}$ higher in energy than the corresponding GM, although all gold atoms are ligated in both cases.

These GMs reveal that ligation can significantly change the stability of the core gold cluster. This effect can be explored by examining the electron density difference of $\mathrm{Au}_{8}^{2+}$ upon ligation. As shown in Figure 7, when Au8c-0 and Au8c-1 are ligated by $4 \mathrm{P}\left(\mathrm{CH}_{3}\right)_{3}$, we observe considerable increase in the electron density for the Au8c-1 core, while for Au8c-0, only minimal changes in the electron density are noted. These changes imply that upon ligation, 3D core clusters offer a better overlap with the phosphine ligands resulting in overall stabilization and change in the stability order of the ligated clusters. Similar effects have been observed in neutral gold clusters, where ligation induced electronic stabilization. ${ }^{115}$ 


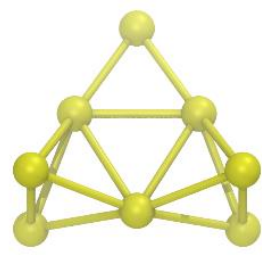

Au8c-0: $0.00 \mathrm{kcal} \mathrm{mol}^{-1}$

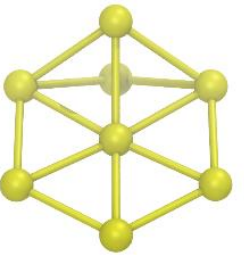

Au8c-1: $4.12 \mathrm{kcal} \mathrm{mol}^{-1}$

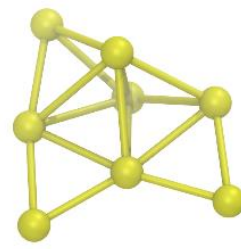

Au8c-2: $6.20 \mathrm{kcal} \mathrm{mol}^{-1}$

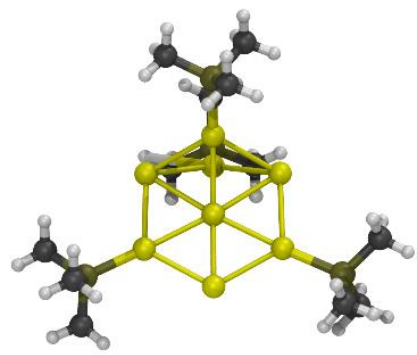

Au8cL4-0: $0.00 \mathrm{kcal}^{\mathrm{mol}^{-1}}$

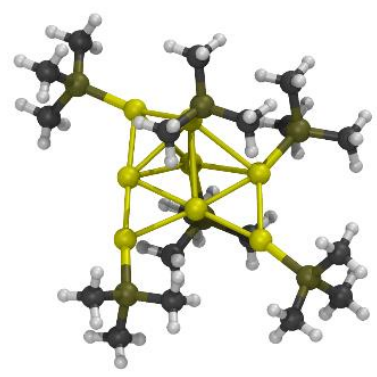

Au8cL6-0

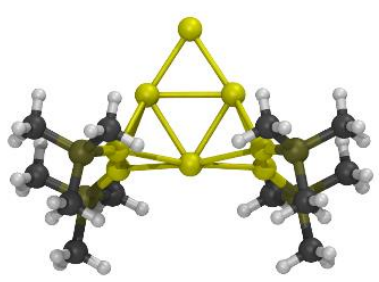

Au8cL4-1: $4.98 \mathrm{kcal} \mathrm{mol}^{-1}$

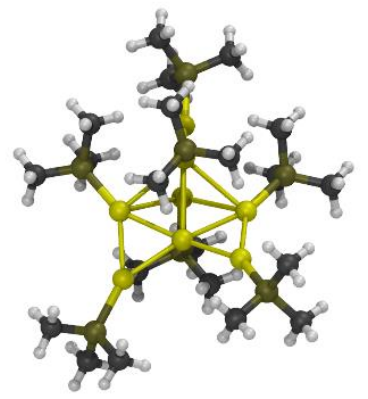

Au8cL7-0

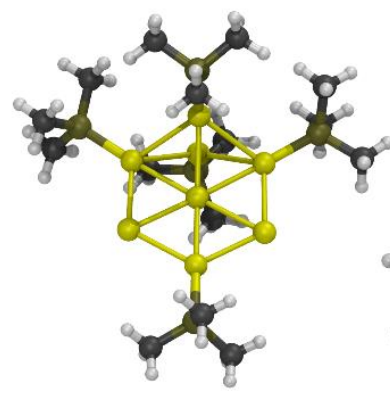

Au8cL5-0: $0.00 \mathrm{kcal} \mathrm{mol}^{-1}$

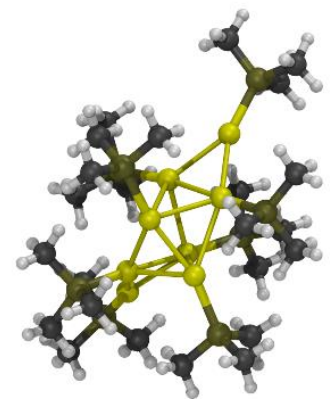

Au8cL8-0: $0.00 \mathrm{kcal} \mathrm{mol}^{-1}$

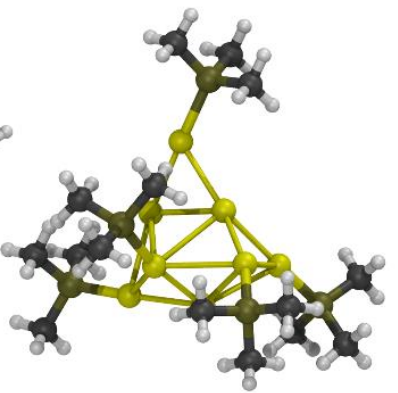

Au8cL5-1: $0.39 \mathrm{kcal} \mathrm{mol}^{-1}$

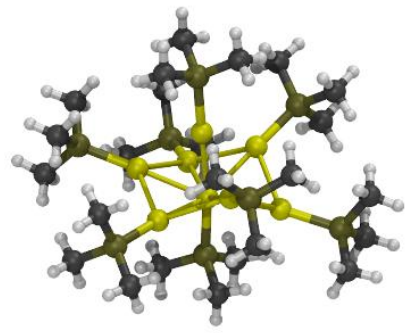

Au8cL8-1: $8.32 \mathrm{kcal} \mathrm{mol}^{-1}$

Figure 6. The GMs of $\mathrm{Au}_{8}^{2+}$ and $\mathrm{Au}_{8} \mathrm{~L}_{n}^{2+}$ found by NWPEsSe. Ligation induces changes in the electronic distribution such that the relative order of stability of the core clusters is completely different than that of the free clusters.

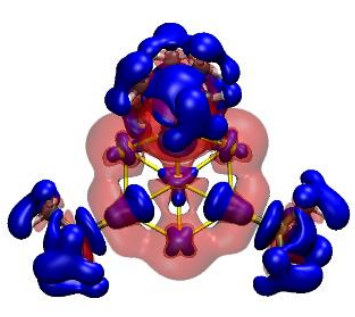

Electron density difference of Au8c-1 upon ligation

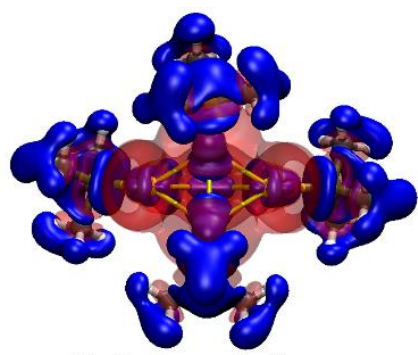

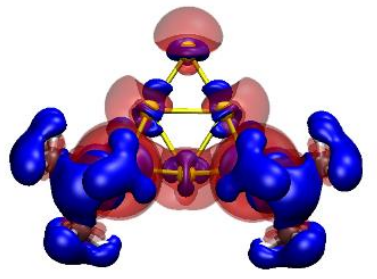

Electron density difference of Au8c-0 upon ligation

Figure 7. Isosurfaces of electron density difference of Au8c-0 and Au8c-1 upon ligated with $4 \mathrm{P}\left(\mathrm{CH}_{3}\right)_{3}$. The difference is defined as $\rho\left[\mathrm{Au}_{8} \mathrm{~L}_{4}^{2+}\right]-\rho\left[\mathrm{Au}_{8}^{2+}\right]-\rho\left[\mathrm{L}_{4}\right]$. The isosurfaces of value +0.001 au and -0.001 au are rendered with transparent red and solid blue, respectively.

\subsection{Surface-Supported Gold Clusters}

Graphene and reducible oxides s are often used as support materials for metal clusters, while performance can be significantly enhanced dues to cooperative effects, ease of separation from products, resistance of high temperature, or modification in their electronic structure. ${ }^{81}$ Here, we also considered gold clusters $\mathrm{Au}_{8}$ supported on three different surfaces: two graphene oxides decorated by epoxy $\left(-\mathrm{O}^{-}\right)$moieties $(\mathbf{G r D}$ where epoxy groups are clustered together and $\mathbf{G r U}$ where epoxy groups are uniformly distributed), and an oxygen-defected rutile $\mathrm{TiO}_{2}(110)$ surface (Rut).
NWPEsSe was used to drive CP2K to carry out the global optimizations at PBE-D3 level of theory using $g_{\max }=100$ to determine the GM for each system. The graphene oxides were modelled by a $1.70 \times 1.68 \mathrm{~nm}$ cell and the rutile $\mathrm{TiO}_{2}(110)$ surface was modelled by a $1.89 \times 1.77 \times 1.09 \mathrm{~nm}$ slab, containing 4 $\mathrm{O}-\mathrm{Ti}-\mathrm{O}$-layers. An oxygen atom from the first $\mathrm{O}-\mathrm{Ti}-\mathrm{O}$ layer was removed to create a defect. Periodic boundary conditions (PBCs) were applied for these cases. In the direction of the surface normal, a $3 \mathrm{~nm}$ vacuum was created and dipole correction was used to remove the artificial field raised by PBCs in this 
direction. ${ }^{116}$ The optimized structures of all supports are shown in Figure 8.

The GM and some LMs of graphene oxide and oxygen-defected rutile $\mathrm{TiO}_{2}(110)$ surface supported $\mathrm{Au}_{8}$ are shown in Figure 9 and 10 , respectively. In gas phase, $\mathrm{Au}_{8}$ tends to be like a flake (Figure 8) due to the relativistic effects, ${ }^{117}$ but they are affected in different ways by the surfaces.

On the graphene oxide supports, we could identify two types of $\mathrm{Au}_{8}$ stationary structures: those that form at least one Au-O bond, and those where no Au-O bonds are formed, see Figure 9. For structures Au8GrD-2, Au8GrD-3, Au8GrU-3, where no $\mathrm{Au}-\mathrm{O}$ forms, the relative stability order for remains the same as in the gas phase, although on GrD the energy difference between Au8-0 and Au8-1 from $6.46 \mathrm{kcal} \mathrm{mol}^{-1}$ reduces to 2.48 $\mathrm{kcal} \mathrm{mol}^{-1}$ (Au8GrD- 2 and Au8GrD-3). This reduction results from the more favorable pure interaction energy between Au81 and GrD, see Supporting Information).

As in the ligated clusters, the formation of covalent bonds between gold cluster and the graphene oxide substantially impacts their electronic structure, and again favors the 3D Au8 structures, as opposed to flakes.

(A)
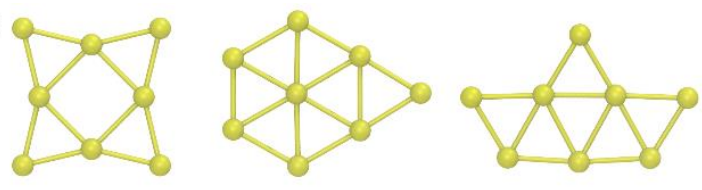

A

(B)

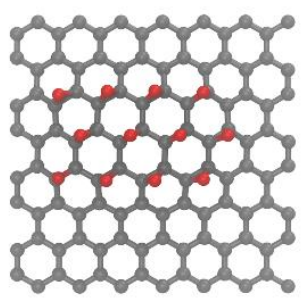

GrD

(C)

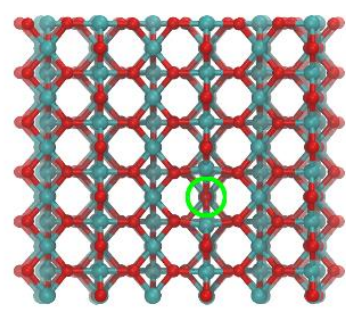

Rut

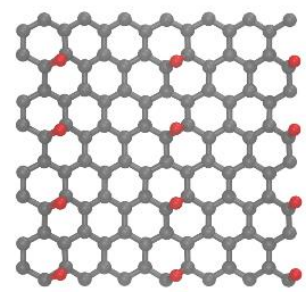

GrU

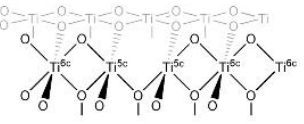

An oxygen atom was

removed from (110)

surface to create a defect

Figure 8. (A) The GM and some LMs of gas phase $\mathrm{Au}_{8}$ found with NWPEsSe+CP2K. (B) Graphene oxides. (C) Oxygen-defected rutile $\mathrm{TiO}_{2}(110)$ surface.

The GMs of graphene oxide-supported $\mathrm{Au}_{8}, \mathbf{A u 8 G r D - 0}$ and Au8GrU-0, both contain two Au-O bonds and have 3D geometries. In the case where epoxy groups from islands, the gold clusters $\mathrm{Au}_{8}$ can land over either oxygen islands or graphenelike areas. In general, landing over the oxide areas is energetically unfavorable. Since the distribution of epoxy or other functional groups can be complicated, ${ }^{118}$ gold cluster structures may be used to fine-tune the physicochemical properties of graphene oxides. For example, anchoring gold clusters on epoxy sites would favor 3D structures instead of flakes, promoting larger interlayer spaces in functionalized graphene membranes and higher separation performance.
Figure 10 suggests that the oxygen-defects also have large electronic effects on the gold clusters. On rutile $\mathrm{TiO}_{2}(110)$ surface, the GM Au8Rut-0, $\mathrm{Au}_{8}$ has a three-dimensional structure, with an $\mathrm{Au}-\mathrm{Au}$ bond pointing towards the oxygen-defect. Substantial flow of electron density towards $\mathrm{Au}_{8}$ is observed, especially on the gold atom occupying the $\mathrm{O}$-vacancy bound to the two fivecoordinated titanium atoms ( $\mathrm{Ti}^{5 \mathrm{c}}$ in Figure 8 and 10). Moreover, the Ti-Au distance is $2.60 \AA$, being smaller than the sum of their covalent radii $2.96 \AA .{ }^{119}$ Both observation indicate the formation of strong Ti-Au bonds. For the LM Au8Rut-1 and Au8Rut-2, $\mathrm{Au}_{8}$ takes a geometry similar to its gas phase $\mathrm{LM}$ Au8-2 and GM Au8-0, respectively. In both cases, one gold atom forms Ti-Au bond at the oxygen-defect. The energy difference becomes much smaller on surface than in gas phase. For example, while Au8-2 is $11.62 \mathrm{kcal} \mathrm{mol}^{-1}$ higher in energy than Au8-0 in the gas phase, on the O-defected rutile $\mathrm{TiO}_{2}(110)$ surface, the difference is reduced to $2.07 \mathrm{kcal} \mathrm{mol}^{-1}$. The electron difference map shows transfer from the adjacent titanium and oxygen atoms, ${ }^{120}$ and on to the cluster. ${ }^{121}$ Therefore, $\mathrm{Au}_{8}$ will become a charge reservoir upon absorption and the PES on the oxygen-defected rutile $\mathrm{TiO}_{2}(110)$ surface becomes flatter than that in gas phase, in compatible with that observation that gold clusters on this surface are very flexible, being liquid-like in dynamics. ${ }^{121}$ Such charge reservoirs play essential roles in heterogeneous catalysis.

To sum up, surfaces can significantly affect the stability order and chemical properties of metal clusters, especially when chemical bonds form or charge transfer occurs. NWPEsSe can be a powerful tool to obtain reliable structures of surface-supported clusters that reveal their electronic and structural diversity.

\subsection{Cluster Assemblies}

Building functional materials often relies on cluster assemblies, $-{ }^{122}$ many of which are stable units functioning as "superatoms". ${ }^{123}$ For example, the electron-rich $\mathrm{Co}_{6} \mathrm{Te}_{8}\left(\mathrm{PEt}_{3}\right)_{6}$ can reversibly donate up to 5 electrons ${ }^{124}$ and fullerene $\mathrm{C}_{60}$ acts as an electron acceptor. Assemblies of these clusters are believed to be precursors to interesting materials. Solids of similar composition, $\left[\mathrm{Co}_{6} \mathrm{Se}_{8}\left(\mathrm{PEt}_{3}\right)_{6}\right]\left[\mathrm{C}_{60}\right]_{2}$ and $\left[\mathrm{Cr}_{6} \mathrm{Te}_{8}\left(\mathrm{PEt}_{3}\right)_{6}\right]\left[\mathrm{C}_{60}\right]_{2}$ have been synthesized and were shown to be good electrical conductors even at room temperature. ${ }^{125}$ We put NWPEsSe to the test to explore: how many $\mathrm{C}_{60}$ can $\mathrm{Co}_{6} \mathrm{Te}_{8}\left(\mathrm{PEt}_{3}\right)_{6}$ accommodate in its first coordination sphere? We examined GMs of $\left[\mathrm{Co}_{6} \mathrm{Te}_{8}\left(\mathrm{PEt}_{3}\right)_{6}\right]\left[\mathrm{C}_{60}\right]_{n}$ for various $n$. Due to the large size of this system $(\sim 3.1 \mathrm{~nm}$ or 1000 atoms), local optimizations were only performed with xTB. For each system, we set $g_{\max }=30$. Initial exploration for smaller $n$ values $(n=5,12,17$ and 19) showed that all $\mathrm{C}_{60}$ superatoms remain in the first coordination shel. For $n=20$, the first coordination sphere becomes full, forming a large cluster of about $3.1 \times 3.1 \times 3.1 \mathrm{~nm}$. The $\mathrm{C}_{60}$ units tend to agglomerate instead of adopting a more uniform distribution, the likely result of the strong dispersion interactions between fullerenes. ${ }^{126}$ For $n=21$, the last $\mathrm{C}_{60}$ starts populating the outer coordination. The composition of these clusters can be adjusted and manipulated in gas phase as molecular devices to trap or transfer electrons. ${ }^{125,127}$ Tailoring these types of materials rely on understanding their bonding and assembly into oligomers and extended structures. However, predicting and exploring their properties largely depends on efficient ways to determine global and local minima and NWPEsSe is a robust and promising tool towards the exploration of very large systems. 
(A)

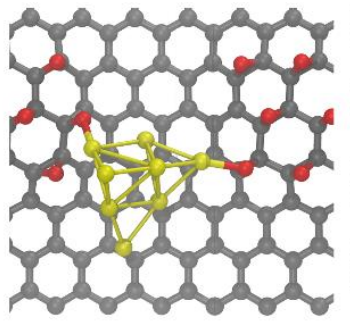

(B)

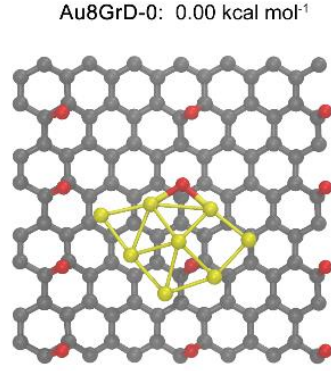

Au8GrU-0: $0.00 \mathrm{kcal} \mathrm{mol}^{-1}$

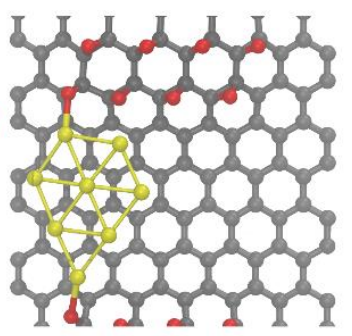

Au8GrD-1: $3.26 \mathrm{kcal} \mathrm{mol}^{-1}$

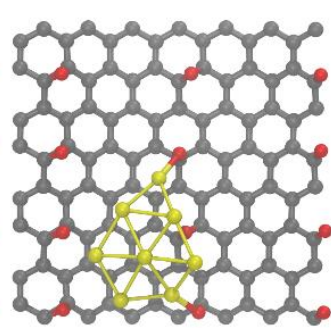

Au8GrU-1: $12.56 \mathrm{kcal} \mathrm{mol}^{-1}$

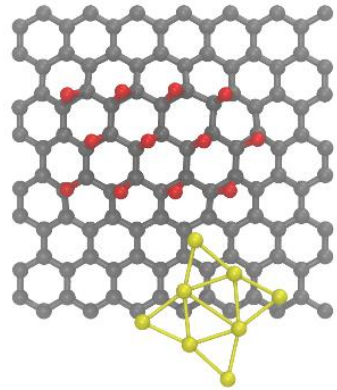

Au8GrD-2: $14.31 \mathrm{kcal} \mathrm{mol}^{-1}$

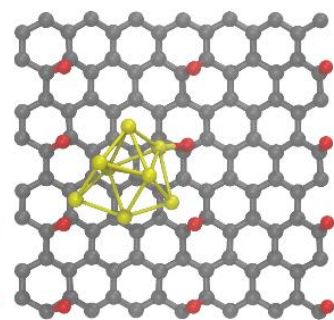

Au8GrU-2: $31.82 \mathrm{kcal} \mathrm{mol}^{-1}$

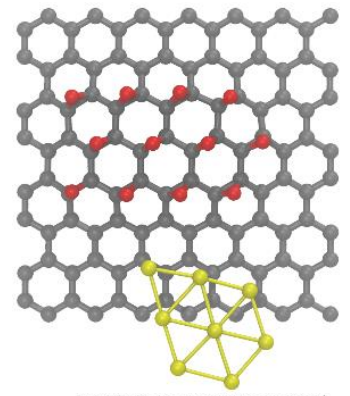

Au8GrD-3: $16.79 \mathrm{kcal} \mathrm{mol}^{-1}$

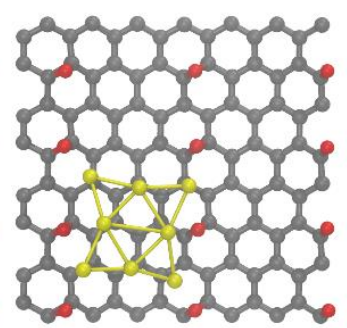

Au8GrD-3: $32.59 \mathrm{kcal} \mathrm{mol}^{-1}$

Figure 9. The GM and some $\mathrm{LMs}$ of $\mathrm{Au}_{8}$ on graphene oxides.
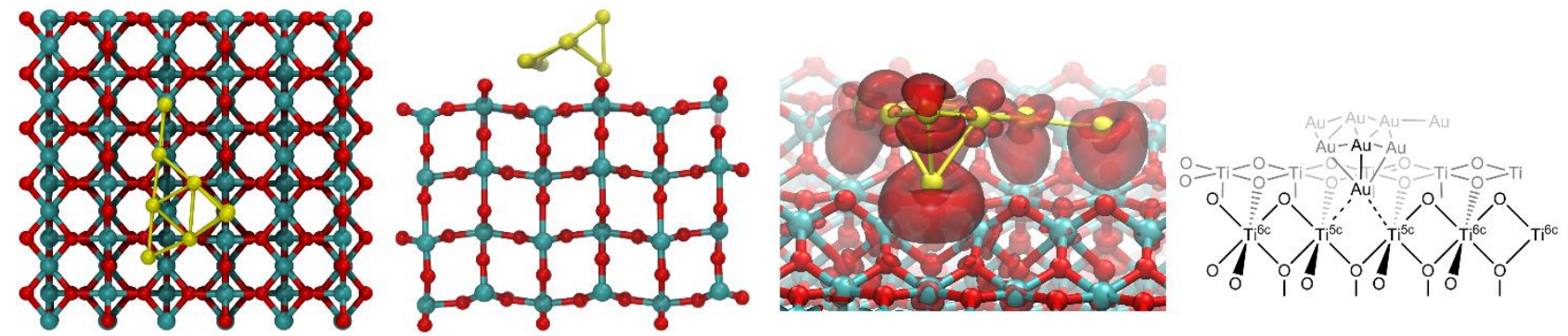

Au8Rut-0: $0.00 \mathrm{kcal} \mathrm{mol}^{-1}$
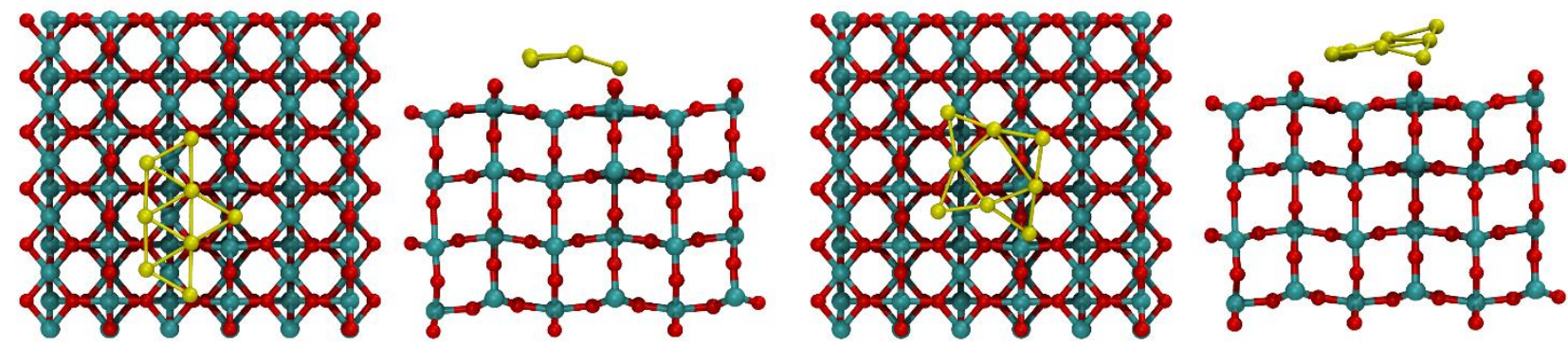

Au8Rut-2: $2.15 \mathrm{kcal} \mathrm{mol}^{-1}$

Figure 10. The GM and some $\mathrm{LMs}_{\mathrm{S}} \mathrm{Au}_{8}$ on oxygen-defected rutile $\mathrm{TiO}_{2}(110)$ surface, and the isosurface (value: +0.001 au) of electron density difference of Au8Rut-0. The difference is defined as $\rho\left[\mathrm{Au}_{8} @ \mathrm{TiO}_{2}\right]-\rho\left[\mathrm{Au}_{8}\right]-\rho\left[\mathrm{TiO}_{2}\right]$. 


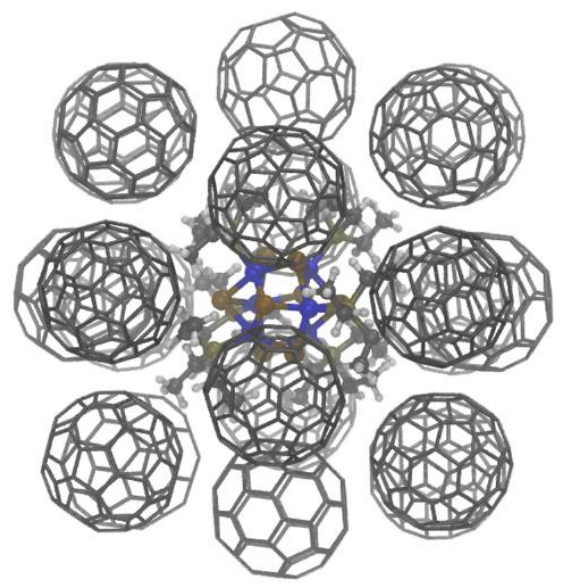

CoreFul20-0

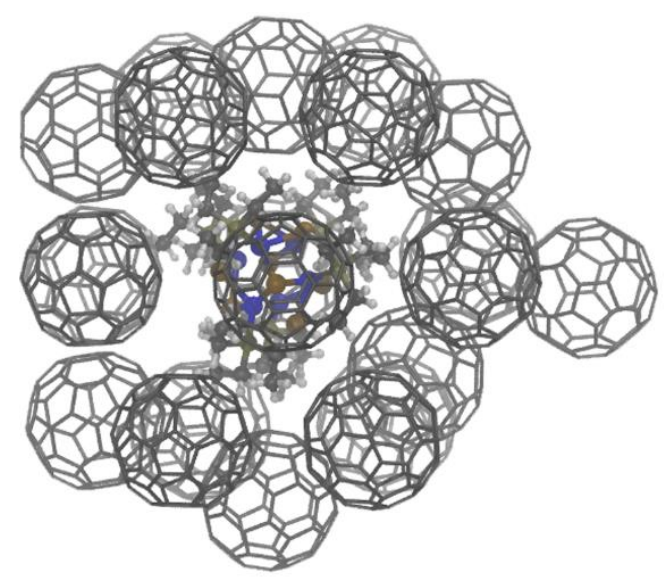

CoreFul21-0

Figure 11. The GMs of $\left[\mathrm{Co}_{6} \mathrm{Te}_{8}\left(\mathrm{PEt}_{3}\right)_{6}\right]\left[\mathrm{C}_{60}\right]_{20 / 21}$.

Table 2. Performance of the global optimizations (SE: semi-empirical).ss

\begin{tabular}{cllll}
\hline Systems & \# atoms & $g_{\max }$ & \# calculations & Resources \\
\hline $\mathrm{Au}_{55}$ & 55 & 3000 & $3000 \mathrm{SE}+30 \mathrm{DFT}$ & 24 CPU cores \\
$\mathrm{Au}_{8} \mathrm{~L}_{n}^{2+}$ & $60 \sim 100$ & 500 & $500 \mathrm{SE}+30 \mathrm{DFT}$ & $24 \mathrm{CPU}$ cores \\
Surface-supported $\mathrm{Au}_{8}$ & $100 \sim 400$ & 100 & $100 \mathrm{DFT}$ & $192 \mathrm{CPU}$ cores \\
{$\left[\mathrm{Co}_{6} \mathrm{Te}_{8}\left(\mathrm{PEt}_{3}\right)_{6}\right]\left[\mathrm{C}_{60}\right]_{n}$} & $\sim 1300$ & 30 & $30 \mathrm{SE}$ & $24 \mathrm{CPU}$ cores \\
\hline
\end{tabular}

\subsection{Summary of Performance}

Table 2 summarizes performance details of NWPEsSe for the systems tested here. The complexity of the global optimization depends on both the number of atoms and the nature of the interaction. NWPEsSe is robust for a wide range of chemical clusters containing up to 1300 atoms: gas phase, ligated or surfacesupported clusters and large molecular assemblies. In practice, homogeneous clusters dominated by short-ranged interactions, like $\mathrm{Au}_{55}$, a large $g_{\text {max }}$ is needed for reliable global optimization. In other cases, a moderate $g_{\text {max }}$ proportional to the number of active degrees of freedoms is sufficient. Although it is not trivial to make a fair comparison of the performance of NWPEsSe with other heuristic ones, Table 2 reveals that reliable global optimizations for a wide range of clusters can be performed with ordinary hardware at an acceptable cost.

\section{Conclusions and Outlook}

In this paper, we discuss a new implementation of the $\mathrm{ABC}$ algorithm tailored for optimization of nanosized clusters. Tested on a variety of systems of varying chemical nature, the new algorithm exhibits excellent performance. In particular, the GMs located for $\mathrm{Au}_{55}$ and $\mathrm{Au}_{8}^{2+}$ are comparable or better than thoseones reported in literature. This algorithm is avialable in NWPEsSe, with interfaces to several computational chemistry programs and can be downloaded at https://store.pnnl.gov/catalogproducts/open-source at no cost. NWPEsSe can also be used as a stand-alone fast generator of complicated structures. The ever-present challenge of global optimization is particularly prominent when confronted with a large number of expensive quantum mechanical calculations or when classical force fields are inaccurate or unavailable. NWPEsSe could be used to overcome this barrier, by screening with a reliable but inexpensive methods, such as xTB available in the code. We are currently working on incorporating data science methods, like machine learning, to improve the global optimization algorithm. ${ }^{128,129}$

\section{ASSOCIATED CONTENT}

Some additional discussions and the structures of clusters mentioned in this paper. This material is available free of charge via the Internet at http://pubs.acs.org.

\section{AUTHOR INFORMATION}

\section{Corresponding Author}

* Corresponding author: Vanda.Glezakou@pnnl.gov

Notes

The authors declare no competing financial interest.

\section{ACKNOWLEDGMENT}

The work described in this publication was performed at Pacific Northwest National Laboratory (PNNL), which is operated by Battelle for the United States Department of Energy (DOE) under Contract DE-AC05-76RL0180. J. Z. and V.-A. G. acknowledge support from DOE, Office of Science, Office of Basic Energy Sciences, Chemical, Geological and Biological Sciences Division and computing resources from PNNL's Research Computing Facility and the National Energy Research Scientific Computing Center.

\section{REFERENCES}

1. Bowlan, J.; Liang, A.; de Heer, W. A., How Metallic are Small Sodium Clusters? Phys. Rev. Lett. 2011, 106 (4), 043401.

2. Niemeyer, M.; Hirsch, K.; Zamudio-Bayer, V.; Langenberg, A.; Vogel, M.; Kossick, M.; Ebrecht, C.; Egashira, 
K.; Terasaki, A.; Möller, T.; v. Issendorff, B.; Lau, J. T., Spin Coupling and Orbital Angular Momentum Quenching in Free Iron Clusters. Phys. Rev. Lett. 2012, 108 (5), 057201.

3. Shibata, T. B., Bruce A.; Zhang, Zhenyuan; Meisel, Dan; Vardeman, Charles F.; Gezelter, J. Daniel, Size-dependent spontaneous alloying of $\mathrm{Au}-\mathrm{Ag}$ nanoparticles. J. Am. Chem. Soc. 2002, 124 (40), 11989-11996.

4. Li, J.; Zhou, S.; Zhang, J.; Schlangen, M.; Usharani, D.; Shaik, S.; Schwarz, H., Mechanistic Variants in Gas-Phase Metal-Oxide Mediated Activation of Methane at Ambient Conditions. J. Am. Chem. Soc. 2016, 138 (35), 11368-11377. 5. Zhao, T.; Wang, Q.; Jena, P., Cluster-Inspired Design of High-Capacity Anode for Li-Ion Batteries. ACS Energy Lett. 2016, 1 (1), 202-208.

6. Heinz, N.; Zhang, J.; Dolg, M., Actinoid(III) Hydration-First Principle Gibbs Energies of Hydration Using High Level Correlation Methods. J. Chem. Theory Comput. 2014, 10 (12), 5593-5598.

7. Zhang, J.; Heinz, N.; Dolg, M., Understanding Lanthanoid(III) Hydration Structure and Kinetics by Insights from Energies and Wave functions. Inorg. Chem. 2014, 53 (14), 77007708 .

8. Basdogan, Y.; Keith, J. A., A Paramedic Treatment for Modeling Explicitly Solvated Chemical Reaction Mechanisms. Chem. Sci. 2018, 9 (24), 5341-5346.

9. Wille, L. T., Minimum-Energy Configurations of Atomic Clusters: New Results Obtained by Simulated Annealing. Chem. Phys. Lett. 1987, 133 (5), 405-410.

10. Li, Z.; Scheraga, H. A., Structure and free energy of complex thermodynamic systems. J. Mol. Struct.: THEOCHEM 1988, 179 (1), 333-352.

11. Wales, D. J.; Scheraga, H. A., Global Optimization of Clusters, Crystals, and Biomolecules. Science 1999, 285 (5432), 1368-1372.

12. Li, Z.; Scheraga, H. A., Monte Carlo-Minimization Approach to the Multiple-Minima Problem in Protein Folding. Proc. Natl. Acad. Sci. U. S. A. 1987, 84, 6611.

13. Wales, D. J.; Doye, J. P. K., Global Optimization by Basin-Hopping and the Lowest Energy Structures of LennardJones Clusters Containing up to 110 Atoms. J. Phys. Chem. A 1997, 101, 5111.

14. White, R. P.; Mayne, H. R., An Investigation of Two Approaches to Basin Hopping Minimization for Atomic and Molecular Clusters. Chem. Phys. Lett. 1998, 289, 463.

15. Call, S. T.; Zubarev, D. Y.; Boldyrev, A. I., Global Minimum Structure Searches via Particle Swarm Optimization. $J$. Comput. Chem. 2007, 28, 1177.

16. Johnston, R. L., Evolving Better Nanoparticles: Genetic Algorithms for Optimising Cluster Geometries. Dalton Trans. 2003, (22), 4193-4207.

17. Hartke, B., Global Geometry Optimization of Clusters Using Genetic Algorithms. J. Phys. Chem. 1993, 97, 9973.

18. Deaven, D. M.; Ho, K. M., Molecular Geometry Optimization with a Genetic Algorithm. Phys. Rev. Lett. 1995, 75, 288.

19. Hartke, B., Global Geometry Optimization of Clusters Using a Growth Strategy Optimized by a Genetic Algorithm. Chem. Phys. Lett. 1995, $240,560$.

20. Daven, D. M.; Tit, N.; Morris, J. R.; Ho, K. M., Structural Optimization of Lennard-Jones Clusters by a Genetic Algorithm. Chem. Phys. Lett. 1996, 256, 195.

21. Kanters, R. P. F.; Donald, K. J., CLUSTER: Searching for Unique Low Energy Minima of Structures Using a Novel Implementation of a Genetic Algorithm. J. Chem. Theory Comput. 2014, 10, 5729.

22. Shayeghi, A.; Götz, D.; Davis, J. B. A.; Schäfer, R.; Johnston, R. L., Pool-BCGA: A Parallelised Generation-Free
Genetic Algorithm for the Ab Initio Global Optimisation of Nanoalloy Clusters. Phys. Chem. Chem. Phys. 2015, 17, 2104. 23. Rabanal-León, W. A.; Tiznado, W.; Osorio, E.; Ferraro, F., Exploring the Potential Energy Surface of Small Lead Clusters Using the Gradient Embedded Genetic Algorithm and an Adequate Treatment of Relativistic Effects. RSC $A d v$. 2018, 8, 145 .

24. Call, S. T.; Zubarev, D. Y.; Boldyrev, A. I., Global Minimum Structure Searches via Particle Swarm Optimization. J. Comput. Chem. 2007, 28 (7), 1177-1186.

25. Shang, C.; Liu, Z.-P., Stochastic Surface Walking Method for Structure Prediction and Pathway Searching. J. Chem. Theory Comput. 2013, 9 (3), 1838-1845.

26. Zhai, H.; Ha, M.-A.; Alexandrova, A. N., AFFCK: Adaptive Force-Field-Assisted Ab Initio Coalescence Kick Method for Global Minimum Search. J. Chem. Theory Comput. 2015, 11 (5), 2385-2393.

27. Addicoat, M. A.; Metha, G. F., Kick: Constraining a Stochastic Search Procedure with Molecullar Fragments. $J$. Comput. Chem. 2009, 30, 57.

28. Bera, P. P.; Schleyer, P. v. R.; Schaefer, H. F., Periodane: A Wealth of Structural Possibilities Revealed by the Kick Procedure. Int. J. Quantum Chem. 2007, 107, 2220.

29. Saunders, M., Stochastic Exploration of Molecular Mechanics Energy Surfaces. Hunting for the Global Minimum. $J$. Am. Chem. Soc. 1987, 109, 3150.

30. Jäger, M.; Schäfer, R.; Johnston, R. L., GIGA: a versatile genetic algorithm for free and supported clusters and nanoparticles in the presence of ligands. Nanoscale 2019, 11 (18), 9042-9052.

31. Heiles, S.; Johnston, R. L., Global Optimization of Clusters Using Electronic Structure Methods. Int. J. Quantum Chem. 2013, 113 (18), 2091-2109.

32. Jäger, M.; Schäfer, R.; Johnston, R. L., First Principles Global Optimization of Metal Clusters and Nanoalloys. $A d v$. Phys.: $X$ 2018, 3 (1), 1516514.

33. Zhang, J.; Dolg, M., ABCluster: the Artificial Bee Colony Algorithm for Cluster Global Optimization. Phys. Chem. Chem. Phys. 2015, 17 (37), 24173-24181.

34. Zhang, J.; Dolg, M., Global Optimization of Clusters of Rigid Molecules Using the Artificial Bee Colony Algorithm. Phys. Chem. Chem. Phys. 2016, 18 (4), 3003-3010.

35. Alexandrova, A. N.; Boldyrev, A. I.; Fu, Y.-J.; Yang, X.; Wang, X.-B.; Wang, L.-S., Structure of the NaxClx+1-(x=14) clusters via ab initio genetic algorithm and photoelectron spectroscopy. The Journal of Chemical Physics 2004, 121 (12), 5709-5719.

36. Barcaro, G.; Aprà, E.; Fortunelli, A., Structure of Ag Clusters Grown on Fs-Defect Sites of an $\mathrm{MgO}\left(\begin{array}{lll}1 & 0 & 0\end{array}\right)$ Surface. Chem. Eur. J. 2007, 13 (22), 6408-6418.

37. Alexandrova, A. N.; Boldyrev, A. I., Search for the Lin0/+1/-1 $(n=5-7)$ Lowest-Energy Structures Using the ab Initio Gradient Embedded Genetic Algorithm (GEGA). Elucidation of the Chemical Bonding in the Lithium Clusters. Journal of Chemical Theory and Computation 2005, 1 (4), 566580 .

38. Davis, J. B. A.; Shayeghi, A.; Horswell, S. L.; Johnston, R. L., The Birmingham parallel genetic algorithm and its application to the direct DFT global optimisation of $\operatorname{IrN}(\mathrm{N}=$ 10-20) clusters. Nanoscale 2015, 7 (33), 14032-14038.

39. Wei, G.-F.; Liu, Z.-P., Subnano Pt Particles from a First-Principles Stochastic Surface Walking Global Search. Journal of Chemical Theory and Computation 2016, 12 (9), 46984706.

40. Shi, H.; Auerbach, S. M.; Ramasubramaniam, A., FirstPrinciples Predictions of Structure-Function Relationships of 
Graphene-Supported Platinum Nanoclusters. The Journal of Physical Chemistry C 2016, 120 (22), 11899-11909.

41. Hussein, H. A.; Davis, J. B. A.; Johnston, R. L., DFT global optimisation of gas-phase and $\mathrm{MgO}$-supported subnanometre AuPd clusters. Physical Chemistry Chemical Physics 2016, 18 (37), 26133-26143.

42. Takasao, G.; Wada, T.; Thakur, A.; Chammingkwan, P.; Terano, M.; Taniike, T., Machine Learning-Aided Structure Determination for TiCl4-Capped $\mathrm{MgCl} 2$ Nanoplate of Heterogeneous Ziegler-Natta Catalyst. ACS Catal. 2019, 9 (3), 2599-2609.

43. Chen, Z.; Jiang, X.; Li, J.; Li, S.; Wang, L., PDECO: Parallel differential evolution for clusters optimization. $J$.

Comput. Chem. 2013, 34 (12), 1046-1059.

44. http://www-wales.ch.cam.ac.uk/GMIN/.

45. Chen, X.; Zhao, Y.-F.; Zhang, Y.-Y.; Li, J., TGMin: An efficient global minimum searching program for free and surface-supported clusters. J. Comput. Chem. 2019, 40 (10), $1105-1112$.

46. Yañez, O.; Báez-Grez, R.; Inostroza, D.; RabanalLeón, W. A.; Pino-Rios, R.; Garza, J.; Tiznado, W., AUTOMATON: A Program That Combines a Probabilistic Cellular Automata and a Genetic Algorithm for Global Minimum Search of Clusters and Molecules. J. Chem. Theory Comput. 2019, 15 (2), 1463-1475.

47. Karaboga, D., An Idea Based on Honey Bee Swarm for Numerical Optimization. Technical Report TR06, Erciyes University: 2005.

48. Liu, Y.; Yang, J.; Cheng, L., Structural Stability and Evolution of Scandium-Doped Silicon Clusters: Evolution of Linked to Encapsulated Structures and Its Influence on the Prediction of Electron Affinities for $\mathrm{ScSi}_{n}(n=4-16)$ Clusters. Inorg. Chem. 2018, 57 (20), 12934-12940.

49. $\quad$ Chen, Y.; Liu, Y.; Li, S.; Yang, J., Theoretical Study on the Growth Behavior and Photoelectron Spectroscopy of Lanthanum-Doped Silicon Clusters $\mathrm{LaSi}_{n}{ }^{0 /-}(n=6-20)$. J. Cluster Sci. 2019, 30 (3), 789-796.

50. He, S.; Yang, J., Study on Structure and Property of Lutetium Introduced Silicon Clusters $\operatorname{LuSi}_{n}(n=3-10)$ and Their Anions with Density Functional Theory. J. Cluster Sci. 2017, 28 (4), 2309-2322.

51. Zhang, Y.; Yang, J.; Cheng, L., Probing Structure, Thermochemistry, Electron Affinity and Magnetic Moment of Erbium-Doped Silicon Clusters $\operatorname{ErSi}_{n}(n=3-10)$ and Their Anions with Density Functional Theory. J. Cluster Sci. 2018, 29 (2), 301-311.

52. Hou, L.; Yang, J.; Liu, Y., Density-Functional Study of the Structures and Properties of Holmium-Doped Silicon Clusters $\operatorname{HoSi}_{n}(n=3-9)$ and Their Anions. J. Mol. Model. 2017, 23 (4), 117.

53. Huang, X.; Yang, J., Probing Structure,

Thermochemistry, Electron Affinity, and Magnetic Moment of Thulium-Doped Silicon Clusters $\operatorname{TmSi}_{n}(n=3-10)$ and Their Anions with Density Functional Theory. J. Mol. Model. 2017, 24 (1), 29.

54. Liu, Y.; Yang, J.; Li, S.; Cheng, L., Structural Growth Pattern of Neutral and Negatively Charged Yttrium-Doped Silicon Clusters $\mathrm{YSi}_{n}{ }^{0 /-}(n=6-20)$ : from Linked to Encapsulated Structures. RSC Adv. 2019, 9 (5), 2731-2739.

55. Feng, Y.; Yang, J.; Liu, Y., Study on the Structures and Properties of Praseodymium-Doped Silicon Clusters $\operatorname{PrSi}_{n}(n=3-$ 9) and Their Anions with Density Functional Schemes. Theor. Chem. Acc. 2016, 135 (12), 258.

56. He, S.; Yang, J., Promethium-Doped Silicon Clusters $\mathrm{PmSi}_{n}(n=3-10)$ and Their Anions: Structures,

Thermochemistry, Electron Affinities and Magnetic Moments.

Theor. Chem. Acc. 2017, 136 (8), 93.
57. Yang, J.; Feng, Y.; Xie, X.; Wu, H.; Liu, Y., Gadolinium-Doped Silicon Clusters $\mathrm{GdSi}_{n}(n=2-9)$ and Their Anions: Structures, Thermochemistry, Electron Affinities, and Magnetic Moments. Theor. Chem. Acc. 2016, 135 (8), 204. 58. Li, H.; Kupiainen-Määttä, O.; Zhang, H.; Zhang, X.; Ge, M., A Molecular-Scale Study on the Role of Lactic Acid in New Particle Formation: Influence of Relative Humidity and Temperature. Atmos. Environ. 2017, 166, 479-487.

59. Li, H.; Zhang, X.; Zhong, J.; Liu, L.; Zhang, H.; Chen, F.; Li, Z.; Li, Q.; Ge, M., The Role of

Hydroxymethanesulfonic Acid in the Initial Stage of New Particle Formation. Atmos. Environ. 2018, 189, 244-251.

60. Ma, X.; Sun, Y.; Huang, Z.; Zhang, Q.; Wang, W., A Density Functional Theory Study of the Molecular Interactions between a Series of Amides and Sulfuric Acid. Chemosphere 2019, 214, 781-790.

61. Zhang, H.; Li, H.; Liu, L.; Zhang, Y.; Zhang, X.; Li, Z., The Potential Role of Malonic Acid in the Atmospheric Sulfuric Acid - Ammonia Clusters Formation. Chemosphere 2018, 203, 26-33.

62. Zhang, H.; Wang, W.; Pi, S.; Liu, L.; Li, H.; Chen, Y.; Zhang, Y.; Zhang, X.; Li, Z., Gas Phase Transformation from Organic Acid to Organic Sulfuric Anhydride: Possibility and Atmospheric Fate in the Initial New Particle Formation. Chemosphere 2018, 212, 504-512.

63. Li, H.; Zhong, J.; Vehkamäki, H.; Kurtén, T.; Wang, W.; Ge, M.; Zhang, S.; Li, Z.; Zhang, X.; Francisco, J. S.; Zeng, X. C., Self-Catalytic Reaction of $\mathrm{SO}_{3}$ and $\mathrm{NH}_{3}$ To Produce Sulfamic Acid and Its Implication to Atmospheric Particle Formation. J. Am. Chem. Soc. 2018, 140 (35), 11020-11028.

64. Kumar, M.; Li, H.; Zhang, X.; Zeng, X. C.; Francisco, J. S., Nitric Acid-Amine Chemistry in the Gas Phase and at the Air-Water Interface. J. Am. Chem. Soc. 2018, 140 (20), 64566466.

65. Liu, L.; Kupiainen-Määttä, O.; Zhang, H.; Li, H.; Zhong, J.; Kurtén, T.; Vehkamäki, H.; Zhang, S.; Zhang, Y.; Ge, M.; Zhang, X.; Li, Z., Clustering Mechanism of Oxocarboxylic Acids Involving Hydration Reaction: Implications for the Atmospheric Models. J. Chem. Phys. 2018, 148 (21), 214303.

66. Ling, J.; Ding, X.; Li, Z.; Yang, J., First-Principles Study of Molecular Clusters Formed by Nitric Acid and Ammonia. J. Phys. Chem. A 2017, 121 (3), 661-668.

67. Myllys, N.; Chee, S.; Olenius, T.; Lawler, M.; Smith, J., Molecular-Level Understanding of Synergistic Effects in Sulfuric Acid-Amine-Ammonia Mixed Clusters. J. Phys. Chem. $A$ 2019, 123 (12), 2420-2425.

68. Hou, G.-L.; Zhang, J.; Valiev, M.; Wang, X.-B., Structures and Energetics of Hydrated Deprotonated cis-Pinonic Acid Anion Clusters and Their Atmospheric Relevance. Phys. Chem. Chem. Phys. 2017, 19 (16), 10676-10684.

69. Zhang, H.; Kupiainen-Määttä, O.; Zhang, X.;

Molinero, V.; Zhang, Y.; Li, Z., The Enhancement Mechanism of Glycolic Acid on the Formation of Atmospheric Sulfuric AcidAmmonia Molecular Clusters. J. Chem. Phys. 2017, 146 (18), 184308.

70. Ahonen, L.; Li, C.; Kubečka, J.; Iyer, S.; Vehkamäki, H.; Petäjä, T.; Kulmala, M.; Hogan Jr, C. J., Ion Mobility-Mass Spectrometry of Iodine Pentoxide-Iodic Acid Hybrid Cluster Anions in Dry and Humidified Atmospheres. J. Phys. Chem. Lett. 2019, 10 (8), 1935-1941.

71. McGrath, M. J.; Olenius, T.; Ortega, I. K.; Loukonen, V.; Paasonen, P.; Kurtén, T.; Kulmala, M.; Vehkamäki, H., Atmospheric Cluster Dynamics Code: a Flexible Method for Solution of the Birth-Death Equations. Atmos. Chem. Phys. 2012, 12 (5), 2345-2355. 
72. Sun, Y.; Liu, S.; Guo, X.; Huang, S., Structural, Magnetic and Electronic Properties of $\mathrm{Cu}_{n} \mathrm{Ni}_{55-n}(n=0-55)$ Nanoparticles: Combination Artificial Bee Colony Algorithm with DFT. Comput. Theor. Chem. 2019, 1154, 11-16.

73. Sengupta, T.; Chung, J. S.; Kang, S. G., Account of Chemical Bonding and Enhanced Reactivity of Vanadium-Doped Rhodium Clusters toward C-H Activation: a DFT Investigation. Phys. Chem. Chem. Phys. 2019.

74. Tan, Q.; Shu, C.; Abbott, J.; Zhao, Q.; Liu, L.; Qu, T.; Chen, Y.; Zhu, H.; Liu, Y.; Wu, G., Highly Dispersed Pd$\mathrm{CeO}_{2}$ Nanoparticles Supported on N-Doped Core-Shell Structured Mesoporous Carbon for Methanol Oxidation in Alkaline Media. ACS Catal. 2019, 9 (7), 6362-6371.

75. Ma, X.; Liu, S.; Huang, S., Hydrogen Adsorption and Dissociation on the TM-Doped (TM=Ti, Nb) Mg 55 Nanoclusters: A DFT Study. Int. J. Hydrogen Energ. 2017, 42 (39), 2479724810.

76. Wu, J.; Wang, L.-W., 2D Framework $\mathrm{C}_{2} \mathrm{~N}$ as a Potential Cathode for Lithium-Sulfur Batteries: an Ab Initio Density Functional Study. J. Mater. Chem. A 2018, 6 (7), 2984-2994. 77. Shao, L.; Wan, K.; Wang, H.; Cui, Y.; Zhao, C.; Lu, J.; Li, X.; Chen, L.; Cui, X.; Wang, X.; Deng, X.; Shi, X.; Wu, Y., A non-conjugated polyethylenimine copolymer-based unorthodox nanoprobe for bioimaging and related mechanism exploration. Biomater. Sci. 2019, 7 (7), 3016-3024.

78. Wu, Z.; Huang, K.; Kuang, X., Dielectric Properties of Pyridine-Ethanol Mixtures: Density Functional Theory and Experiments. RSC Adv. 2016, 6 (70), 66007-66010.

79. Cao, X.; Heinz, N.; Zhang, J.; Dolg, M., The First Water Coordination Sphere of Lanthanide(III) Motexafins (LnMotex $^{2+}, \mathrm{Ln}=\mathrm{La}, \mathrm{Gd}, \mathrm{Lu}$ ) and its Effects on Structures, Reduction Potentials and UV-Vis Absorption Spectra. Theoretical Studies. Phys. Chem. Chem. Phys. 2017, 19 (30), 20160-20171.

80. Wu, Z.; Huang, K., Abnormal Characteristics of Binary Molecular Clusters in DMSO-Ethanol Mixtures under External Electric Fields. Mol. Phys. 2018, 116 (9), 1198-1207.

81. Guzman, J.; Gates, B. C., Supported Molecular Catalysts: Metal Complexes and Clusters on Oxides and Zeolites. Dalton Trans. 2003, (17), 3303-3318.

82. Copéret, C.; Comas-Vives, A.; Conley, M. P.; Estes, D. P.; Fedorov, A.; Mougel, V.; Nagae, H.; Núñez-Zarur, F.; Zhizhko, P. A., Surface Organometallic and Coordination Chemistry toward Single-Site Heterogeneous Catalysts: Strategies, Methods, Structures, and Activities. Chem. Rev. 2016, $116(2), 323-421$.

83. Gupta, R. P., Lattice Relaxation at a Metal Surface. Phys. Rev. B 1981, 23 (12), 6265-6270.

84. Rossi, G.; Rapallo, A.; Mottet, C.; Fortunelli, A.; Baletto, F.; Ferrando, R., Magic Polyicosahedral Core-Shell Clusters. Phys. Rev. Lett. 2004, 93 (10), 105503.

85. Barber, C. B.; Dobkin, D. P.; Huhdanpaa, H., The Quickhull Algorithm for Convex Hulls. ACM Trans. Math. Softw. 1996, 22 (4), 469-483.

86. Thomson, J. J., XXIV. On the Structure of the Atom: an Investigation of the Stability and Periods of Oscillation of a Number of Corpuscles Arranged at Equal Intervals around the Circumference of a Circle; with Application of the Results to the Theory of Atomic Structure. Philos. Mag. 1904, 7 (39), 237-265. 87. Liu, D. C.; Nocedal, J., On the Limited Memory BFGS Method for Large Scale Optimization. Math. Program. 1989, 45 (1), 503-528.

88. Fan, H.-Y.; Lampinen, J., A Trigonometric Mutation Operation to Differential Evolution. J. Global Optim. 2003, 27 (1), 105-129.

89. Das, S.; Suganthan, P. N., Differential Evolution: A Survey of the State-of-the-Art. IEEE Transactions on Evolutionary Computation 2011, 15 (1), 4-31.
90. Gao, W.; Liu, S., Improved artificial bee colony algorithm for global optimization. Inform. Process. Lett. 2011, 111 (17), 871-882.

91. Bonabeau, E.; Dorigo, M.; Marco, D. d. R. D. F.; Theraulaz, G.; Théraulaz, G., Swarm intelligence: from natural to artificial systems. Oxford university press: 1999.

92. Grimme, S.; Bannwarth, C.; Shushkov, P., A Robust and Accurate Tight-Binding Quantum Chemical Method for Structures, Vibrational Frequencies, and Noncovalent Interactions of Large Molecular Systems Parametrized for All spd-Block Elements $(Z=1-86)$. J. Chem. Theory Comput. 2017, 13 (5), 1989-2009.

93. Bannwarth, C.; Ehlert, S.; Grimme, S., GFN2-xTBAn Accurate and Broadly Parametrized Self-Consistent TightBinding Quantum Chemical Method with Multipole Electrostatics and Density-Dependent Dispersion Contributions. J. Chem. Theory Comput. 2019, 15 (3), 1652-1671.

94. VandeVondele, J.; Krack, M.; Mohamed, F.; Parrinello, M.; Chassaing, T.; Hutter, J., Quickstep: Fast and Accurate Density Functional Calculations Using a Mixed Gaussian and Plane Waves Approach. Comput. Phys. Commun. 2005, 167 (2), 103-128.

95. http://www.cp2k.org/.

96. Perdew, J. P.; Burke, K.; Ernzerhof, M., Generalized Gradient Approximation Made Simple [Phys. Rev. Lett. 77, 3865 (1996)]. Phys. Rev. Lett. 1997, 78 (7), 1396-1396.

97. Grimme, S.; Antony, J.; Ehrlich, S.; Krieg, H., A Consistent and Accurate $\mathrm{Ab}$ Initio Parametrization of Density Functional Dispersion Correction (DFT-D) for the 94 Elements H-Pu. J. Chem. Phys. 2010, 132 (15), 154104.

98. VandeVondele, J.; Hutter, J., Gaussian Basis Sets for Accurate Calculations on Molecular Systems in Gas and Condensed Phases. J. Chem. Phys. 2007, 127 (11), 114105.

99. Lippert, B. G.; Parrinello, J. H.; Michele, A Hybrid Gaussian and Plane Wave Density Functional Scheme. Mol. Phys. 1997, 92 (3), 477-488.

100. Goedecker, S.; Teter, M.; Hutter, J., Separable DualSpace Gaussian Pseudopotentials. Phys. Rev. B 1996, 54 (3), 1703-1710.

101. Krack, M., Pseudopotentials for $\mathrm{H}$ to $\mathrm{Kr}$ Optimized for Gradient-Corrected Exchange-Correlation Functionals. Theor. Chem. Acc. 2005, 114 (1), 145-152.

102. Daniel, M.-C.; Astruc, D., Gold Nanoparticles: Assembly, Supramolecular Chemistry, Quantum-Size-Related Properties, and Applications toward Biology, Catalysis, and Nanotechnology. Chem. Rev. 2004, 104 (1), 293-346.

103. Assadollahzadeh, B.; Schwerdtfeger, P., A Systematic Search for Minimum Structures of Small Gold Clusters $\mathrm{Au}_{n}(n=$ 2-20) and Their Electronic Properties. J. Chem. Phys. 2009, 131 (6), 064306.

104. Van den Bossche, M., DFTB-Assisted Global Structure Optimization of 13- and 55-Atom Late Transition Metal Clusters. J. Phys. Chem. A 2019, 123 (13), 3038-3045.

105. Tarrat, N.; Rapacioli, M.; Cuny, J.; Morillo, J.; Heully, J.-L.; Spiegelman, F., Global Optimization of Neutral and Charged 20- and 55-atom Silver and Gold Clusters at the DFTB Level. Comput. Theor. Chem. 2017, 1107, 102-114.

106. Zhang, Y.; Yang, W., Comment on “Generalized Gradient Approximation Made Simple". Phys. Rev. Lett. 1998, 80 (4), 890-890.

107. Perdew, J. P.; Ruzsinszky, A.; Csonka, G. I.; Vydrov, O. A.; Scuseria, G. E.; Constantin, L. A.; Zhou, X.; Burke, K., Restoring the Density-Gradient Expansion for Exchange in Solids and Surfaces. Phys. Rev. Lett. 2008, 100 (13), 136406.

108. Huang, W.; Ji, M.; Dong, C.-D.; Gu, X.; Wang, L.M.; Gong, X. G.; Wang, L.-S., Relativistic Effects and the 
Unique Low-Symmetry Structures of Gold Nanoclusters. $A C S$ Nano 2008, 2 (5), 897-904.

109. Wang, Z. W.; Palmer, R. E., Experimental Evidence for Fluctuating, Chiral-Type Au55 Clusters by Direct Atomic Imaging. Nano Lett. 2012, 12 (11), 5510-5514.

110. Schrader, I.; Warneke, J.; Backenköhler, J.; Kunz, S., Functionalization of Platinum Nanoparticles with 1-Proline: Simultaneous Enhancements of Catalytic Activity and Selectivity. J. Am. Chem. Soc. 2015, 137 (2), 905-912.

111. Chen, G.; Xu, C.; Huang, X.; Ye, J.; Gu, L.; Li, G.; Tang, Z.; Wu, B.; Yang, H.; Zhao, Z.; Zhou, Z.; Fu, G.; Zheng, N., Interfacial Electronic Effects Control the Reaction Selectivity of Platinum Catalysts. Nat. Mater. 2016, 15, 564.

112. Laskin, J.; Johnson, G. E.; Warneke, J.; Prabhakaran, V., From Isolated Ions to Multilayer Functional Materials Using Ion Soft Landing. Angewandte Chemie International Edition 2018, 57 (50), 16270-16284.

113. Parrish, K. A.; King, M.; Ligare, M. R.; Johnson, G. E.; Hernández, H., Role of sterics in phosphine-ligated gold clusters. Physical Chemistry Chemical Physics 2019, 21 (4), 1689-1699.

114. Hong, S.; Shafai, G.; Bertino, M.; Rahman, T. S., Toward an Understanding of Ligand Selectivity in Nanocluster Synthesis. J. Phys. Chem. C 2011, 115 (30), 14478-14487. 115. Shafai, G.; Hong, S.; Bertino, M.; Rahman, T. S., Effect of Ligands on the Geometric and Electronic Structure of $\mathrm{Au}_{13}$ Clusters. J. Phys. Chem. C 2009, 113 (28), 12072-12078. 116. Bengtsson, L., Dipole Correction for Surface Supercell Calculations. Phys. Rev. B 1999, 59 (19), 12301-12304.

117. Häkkinen, H.; Moseler, M.; Landman, U., Bonding in $\mathrm{Cu}, \mathrm{Ag}$, and Au Clusters: Relativistic Effects, Trends, and Surprises. Phys. Rev. Lett. 2002, 89 (3), 033401.

118. Nguyen, M.-T.; Erni, R.; Passerone, D., TwoDimensional Nucleation and Growth Mechanism Explaining Graphene Oxide Structures. Phys. Rev. B 2012, 86 (11), 115406. 119. Cordero, B.; Gómez, V.; Platero-Prats, A. E.; Revés, M.; Echeverría, J.; Cremades, E.; Barragán, F.; Alvarez, S., Covalent Radii Revisited. Dalton Trans. 2008, (21), 2832-2838. 120. Chrétien, S.; Metiu, H., O2 Evolution on a Clean Partially Reduced Rutile $\mathrm{TiO}_{2}(110)$ Surface and on the Same
Surface Precovered with $\mathrm{Au}_{1}$ and $\mathrm{Au}_{2}$ : The Importance of Spin Conservation. J. Chem. Phys. 2008, 129 (7), 074705.

121. Wang, Y.-G.; Yoon, Y.; Glezakou, V.-A.; Li, J.; Rousseau, R., The Role of Reducible Oxide-Metal Cluster Charge Transfer in Catalytic Processes: New Insights on the Catalytic Mechanism of $\mathrm{CO}$ Oxidation on $\mathrm{Au} / \mathrm{TiO}_{2}$ from $\mathrm{A} b$ Initio Molecular Dynamics. J. Am. Chem. Soc. 2013, 135 (29), 10673 10683.

122. Khanna, S. N.; Jena, P., Assembling Crystals from Clusters. Phys. Rev. Lett. 1992, 69 (11), 1664-1667.

123. Doud, E. A.; Voevodin, A.; Hochuli, T. J.;

Champsaur, A. M.; Nuckolls, C.; Roy, X., Superatoms in materials science. Nature Reviews Materials 2020.

124. Turkiewicz, A.; Paley, D. W.; Besara, T.; Elbaz, G.; Pinkard, A.; Siegrist, T.; Roy, X., Assembling Hierarchical Cluster Solids with Atomic Precision. J. Am. Chem. Soc. 2014, 136 (45), 15873-15876.

125. Roy, X.; Lee, C.-H.; Crowther, A. C.; Schenck, C. L.; Besara, T.; Lalancette, R. A.; Siegrist, T.; Stephens, P. W.; Brus, L. E.; Kim, P.; Steigerwald, M. L.; Nuckolls, C., Nanoscale Atoms in Solid-State Chemistry. Science 2013, 341 (6142), 157-160.

126. Zhang, J.; Dolg, M., Dispersion Interaction Stabilizes Sterically Hindered Double Fullerenes. Chem. Eur. J. 2014, 20 (43), 13909-13912.

127. Shubina, T. E.; Sharapa, D. I.; Schubert, C.; Zahn, D.; Halik, M.; Keller, P. A.; Pyne, S. G.; Jennepalli, S.; Guldi, D. M.; Clark, T., Fullerene Van der Waals Oligomers as Electron Traps. J. Am. Chem. Soc. 2014, 136 (31), 10890-10893.

128. Meldgaard, S. A.; Kolsbjerg, E. L.; Hammer, B., Machine Learning Enhanced Global Optimization by Clustering Local Environments to Enable Bundled Atomic Energies. $J$. Chem. Phys. 2018, 149 (13), 134104.

129. Jacobsen, T. L.; Jørgensen, M. S.; Hammer, B., On-theFly Machine Learning of Atomic Potential in Density Functional Theory Structure Optimization. Phys. Rev. Lett. 2018, 120 (2), 026102 . 